\title{
Reverse Thinking of Aggregation-Induced Emission Principle: Amplifying Molecular Motions to Boost Photothermal Efficiency of Nanofibers
}

Haoxuan Li ${ }^{a, b, c}$, Haifei Wen ${ }^{a}$, Zhijun Zhang ${ }^{a, b}$, Nan Song ${ }^{a, b, c}$, Dong Wang ${ }^{* a}$, Ben Zhong Tang ${ }^{* c}$

a Centre for AIE Research, College of Material Science and Engineering, Shenzhen University, Shenzhen 518061, P. R. China

E-mail: wangd@szu.edu.cn

b Key Laboratory of Optoelectronic Devices and Systems of Ministry of Education and Guangdong Province, College of Optoelectronic Engineering, Shenzhen University, Shenzhen 518061, P. R. China

c Department of Chemistry, Hong Kong Branch of Chinese National Engineering Research Center for Tissue Restoration and Reconstruction, Institute of Molecular Functional Materials, The Hong Kong University of Science and Technology Clear Water Bay, Kowloon, Hong Kong 999077, China

E-mail: tangbenz@ust.hk 


\begin{abstract}
Development of efficient photothermal nanofibers is of vital importance, but remaining a big challenge. Herein, with reverse thinking of aggregation-induced emission (AIE) principle, we demonstrate an ingenious and universal protocol for amplifying molecular motions to boost photothermal efficiency of nanofibers. Core-shell nanofibers having the olive oil solution of AIE-active molecules as the core surrounded by PVDF-HFP shell were constructed by coaxial electrospinning. The molecularly dissolved state of AIE-active molecules allows them to freely rotate and/or vibrate in nanofibers upon photoexcitation and thus significantly elevates the proportion of non-radiative energy dissipation, affording impressive heat-generating efficiency. Photothermal evaluation shows that the core-shell nanofibers with excellent durability can reach up to $22.36 \%$ of photothermal conversion efficiency, which is 26 -fold as the non-core-shell counterpart. Noticeably, such core-shell nanofiber can be used for photothermal textiles and solar steam generation $\left(1.52 \mathrm{~kg} \mathrm{~m}^{-1} \mathrm{~h}^{-1}\right.$ under 1 sun) induced by natural sunlight with green and carbon-zero emission.
\end{abstract}

Keywords: Aggregation-induced emission; Molecular motion; Non-radiative decay; Solar energy; Core-shell nanofibers; Solar steam generation. 


\section{Table of Contents}
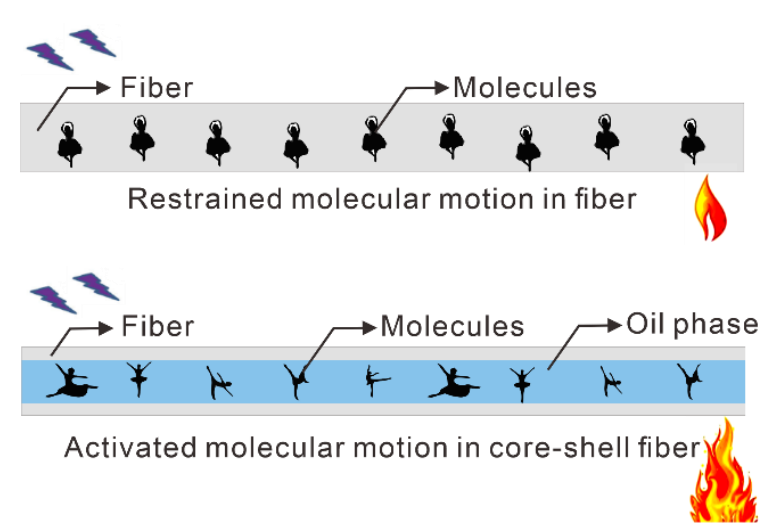

With reverse thinking of aggregation-induced emission (AIE) principle, core-shell nanofiber is facilely fabricated and proven significantly powerful to activate molecular motion for boosting photothermal efficiency of AIE-active molecules in nanofibers. 
Hybrid nanofibers comprising functional molecules have captivated much scientific interest by the virtue of their prominent application potential in smart textiles, wearable device and biomedicine. ${ }^{1-5}$ Of particular interest is photothermal nanofibers that are capable of absorbing light energy and transfer it to heat, affording extensive uses in various applications ranging from the solar steam generation, smart clothing, thermal management to cancer therapy. ${ }^{6-8}$ Finely engineering nanofibers with photothermal function has consequently become one of the most intensive research topics during recent years. However, the state-of-the-art development of this area is not able to satisfy the requirements for practical applications, mainly owing to the insufficient photothermal efficiency of those exploited nanofibers, ${ }^{9,10}$ which essentially originates from the inherent incompatibility between working mechanism of photothermal molecular species and the nature of nanofiber. It has been demonstrated that molecular motion can facilitate thermal expansion upon light absorption by promoting energy dissipation via non-radiative decay pathways. ${ }^{11-13}$ After incorporating of photothermal molecular species in solid nanofiber, the molecular motion, however, is inevitably to some extent restricted by bulky intermolecular steric hindrance, accordingly reducing the photothermal outcomes. Therefore, amplifying molecular motions in nanofiber to boost its photothermal efficiency is of vital importance but challenging task.

Given the circumstances, it is certainly believed that simultaneously manipulating architectures of both photothermal molecular specie and nanofiber is supremely imperative. Considering the significance of molecular motion for improving photothermal efficiency, the exploitation of dyes structurally bearing sufficient molecular rotors and/or vibrators could be highly vital. As such a family of compounds, aggregation-induced emission luminogens (AIEgens) would be ideal candidates. AIE refers to a unique phenomenon that some fluorophores with propeller-like twisted conformation exhibit vigorous intramolecular motions as molecularly dissolved in solvent upon photoexcitation, enabling the energy of the excited state to be consumed mainly through non-radiative decay pathways, but they emit intensively in aggregates due to the restriction of intramolecular motions. ${ }^{14-19}$ Evidently, keeping AIEgens as single 
molecule state is particularly critical to maximally retain the molecular motions and further boost the photothermal capability. In the related context, from the view of nanofiber, the utilization of core-shell nanofibers generated by coaxial electrospinning is considered to be promising for realizing maximal molecular motions of AIEgens, since some liquids such as oil can be readily stretched into long fibers by coaxial electrospinning with immiscible and spinnable solution. ${ }^{20-24}$

In this contribution, the subtle construction of core-shell nanofiber with AIE moleculescontaining oil as core phase and polymer as shell is demonstrated. The combination of AIE-active molecules with core-shell nanofibrous structure maximally retains the molecular motions, weakening the radiation decay. It was observed that these novel core-shell nanofibers demonstrate significantly boosted photothermal efficiency.

By means of this protocol, abundant moieties of AIEgen can freely rotate and/or vibrate in nanofibers, and thus enable non-radiative decay pathways to dominate the excited-state energy dissipation (Figure 1A), making the heat generation significantly powerful. Coaxial electrospinning technique with AIEgen oil solution as coaxial inner layer and poly(vinylidene fluoride-co-hexafluoropropylene) (PVDF-HFP) polymer solution as coaxial outer layer was utilized in the fabrication of nanofibers (Figure 1B). PVDF-HFP is one of widely used electrospun materials benefiting from its excellent stability and spinnability. As illustrated in Figure 1C, the oil phase (dark green) was wrapped absolutely by PVDF-HFP phase (colorless), suggesting that the oil phase can be stretched with polymer via contact friction during rapid elongation of electrospinning. In the nanofibers with precise core-shell architecture, the AIEgen oil solution was encapsulated in the core surrounded by polymer shell layer to avert leakage (Figure 1D). ${ }^{25}$ As a proof of concept, AIE-active molecule BPBBT $^{26,27}$ was used as a model system because of its superior rotation and/or vibration in oil phase (Figure 1D and Figure S1). 


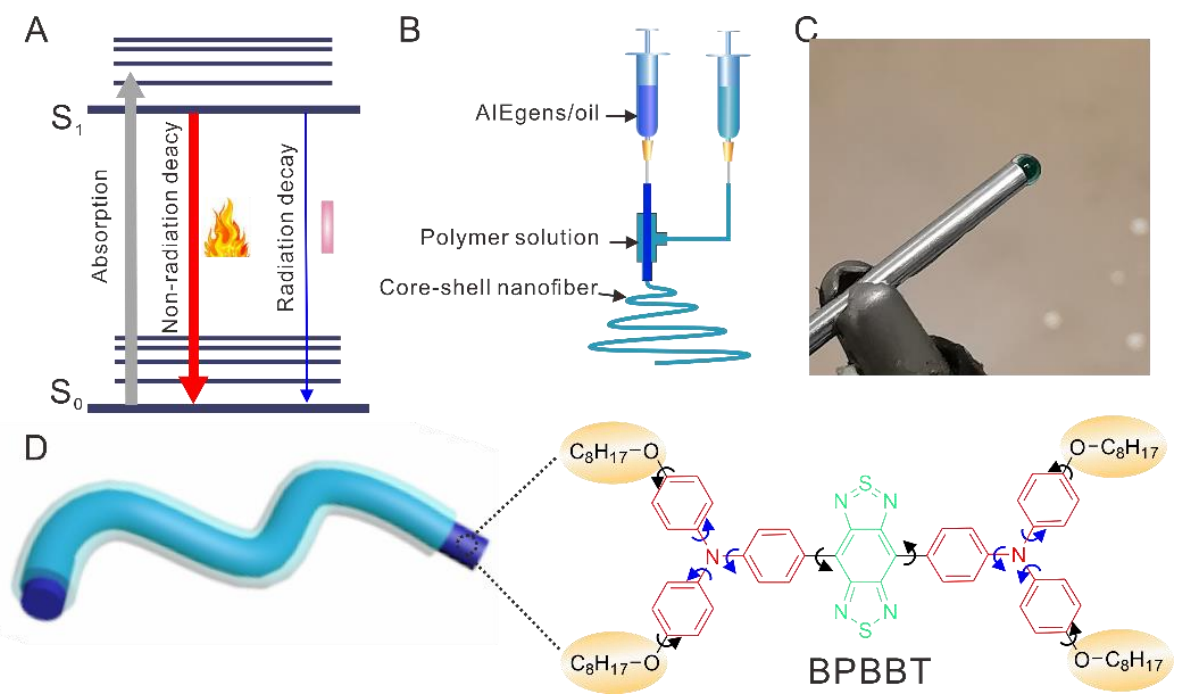

Figure 1. (A) Jablonski diagram illustrating excited-state energy dissipations of AIEgen in the constructed nanofibers. (B) Schematic setup of coaxial electrospinning. (C) Photograph image of the core-shell needle, oil phase (dark-green) was successfully wrapped by polymer phase (colorless) at the needle tip. (D) Schematic structure of a core-shell nanofiber and molecular motion of BPBBT in oil phase.

In the preliminary investigation, we estimated the photothermal capacity of BPBBT in solution and solid state. $20 \mathrm{mg}$ of BPBBT was dissolved in $10 \mathrm{~mL}$ of olive oil as solution state, $12 \mathrm{mg}$ of BPBBT dissolved in $10 \mathrm{~mL}$ of PVDF-HFP $(1.8 \mathrm{~g})$ solution in DMF/THF (7:3), and then electrospun into nanofiber as control sample (Figure S2A). For the construction of the core-shell nanofibers, BPBBT olive oil solution was employed as the core phase with pumping rate at $0.9 \mathrm{~mL} / \mathrm{h}$, and pure PVDF-HFP solution in DMF/THF $(7: 3)(18 \%, \mathrm{~m} / \mathrm{v})$ mixture was utilized as the shell phase with pumping rate at $1.7 \mathrm{~mL} / \mathrm{h}$., such core-shell nanofiber was named as CS-3. BPBBT possesses strong and broad absorption range from 600 to $900 \mathrm{~nm}$ with a maximum at $780 \mathrm{~nm}$ (Figure 2A), which is beneficial to absorb light energy of sunlight. The absorption of BPBBT nanofiber has a blue shift, the reason is maybe the generation of hydrogen bond between $\mathrm{H}$ atom of BPBBT and $\mathrm{F}$ atom of PVDF-HFP, restricted the motion of molecules. The quantum yield (QY) of BPBBT nanofiber is high as 2.9\%, while that of BPBBT/oil solution and BPBBT CS-3 is only $0.2 \%$ (Figure $2 \mathrm{~B}$ ), suggesting that the molecule motion of BPBBT in nanofiber was significantly suppressed by hydrogen bond and polymer chains, resulting in 
boosted radiation decay. On the other hand, the molecules motion of BPBBT is activated in core-shell fiber (CS-3), due to the present of oil, as a result of weakened radiation decay. By exposure to simulated sunlight irradiation with $1 \mathrm{~kW} / \mathrm{m}^{2}$ of power density (1 sun), the temperature of BPBBT/oil and CS-3 reached up to 61.1 and $56.1{ }^{\circ} \mathrm{C}$, while that of $\mathrm{BPBBT}$ nanofiber and BPBBT powder only increased to 40.4 and $38.2{ }^{\circ} \mathrm{C}$ (Figure 2C). These results indicate that the core-shell nanofiber with BPBBT/oil as core phase is an ingenious strategy to activate molecules motion and boost the photothermal efficiency of nanofiber.
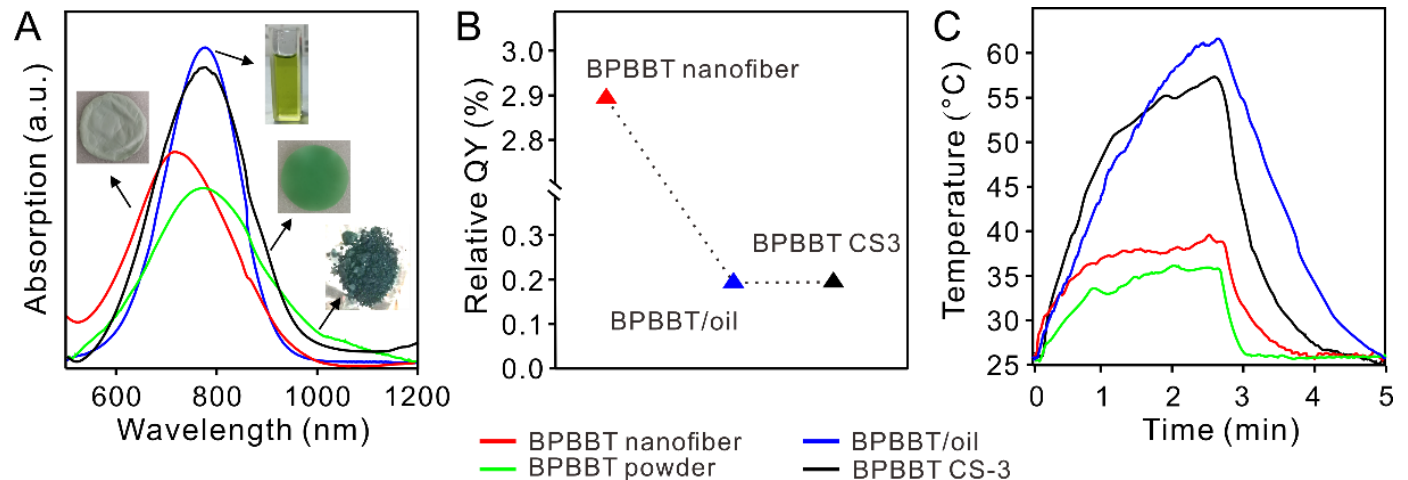

Figure 2. (A) Absorption spectra and (B) relative QY of BPBBT powder, BPBBT nanofiber, BPBBT/oil solution and BPBBT CS-3. (C) Plots showing the changes of temperature of BPBBT powder, BPBBT nanofiber, BPBBT/oil solution and BPBBT CS-3 as they were exposed under 1 sun irradiation.

BPBBT/oil solution was employed as the core phase with pumping rate at 0.3 and $0.6 \mathrm{~mL} / \mathrm{h}$, and PVDF-HFP solution in DMF/THF (7:3) mixture was utilized as the shell phase with pumping rate at $1.7 \mathrm{~mL} / \mathrm{h}$. As illustrated in Figure S2B and C, uniform and continuous nanofibers (namely CS-1 and CS-2) with core-shell structure were successfully fabricated (Table S1). When the nonwoven mat of CS-1 and CS-2 was illuminated under solar irradiation at a power density of 1 sun, the temperature of the mat rise to 46.8 and $50.3^{\circ} \mathrm{C}$, which is higher than BPBBT nanofiber $\left(40.4^{\circ} \mathrm{C}\right)$ (Figure S3A and B). Notably, the BPBBT content in CS-1 and CS-2 was $0.2 \%$ and $0.4 \%$, while that in BPBBT nanofiber is high as $0.6 \%$, suggesting that the photothermal efficiency of BPBBT molecules in core-shell fiber was significantly boosted. In addition, the CS-3 nanofibrous mats exhibited green color, where the BPBBT content is $0.6 \%$ (Figure $3 \mathrm{~A}$, 
Figure S2D). Within irradiation of 1 sun, the temperatures of CS-3 could reach up to $56.1{ }^{\circ} \mathrm{C}$, which are ultrahigh than BPBBT nanofibers. Such excellent photothermal performance should be attributed to effective molecular motion of BPBBT molecules in core phase.
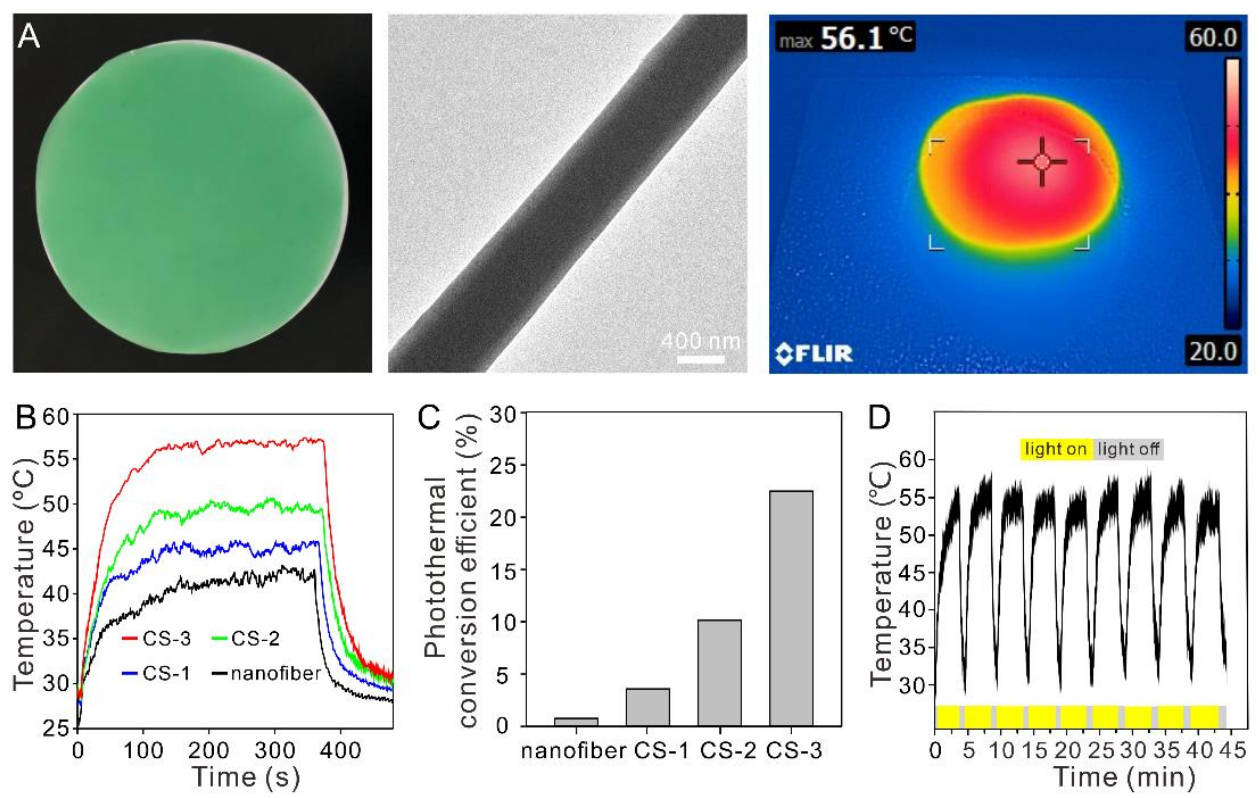

Figure 3. (A) (Left) photographs, (middle)TEM image, and (right) infrared image of the core-shell nanofibrous mats of CS-3. (B) The temperature changes and (C) the photothermal conversion efficiencies of the BPBBT/PVDF-HFP nanofibrous mat and those core-shell nanofibrous mats as they were exposed under 1 sun irradiation. (D) Plots showing the changes of temperature in the mat of CS-3 upon repeated irradiation by 1 sun.

We then investigated the photothermal conversion efficiency of BPBBT molecules in core-shell nanofiber and their durability. The photothermal conversion efficiency could be calculated by following equation: ${ }^{28}$

$$
Q=c m \Delta T
$$

Where $Q, c, m, \Delta T$ are the total heat, specific heat (PVDF-HFP:1.12 $\mathrm{J} \mathrm{g}^{-1} \mathrm{~K}^{-1}$ and Oliver oil:1.96 $\mathrm{J} \mathrm{g}^{-1} \mathrm{~K}^{-1}$ ), mass and the change of temperature, respectively. The total energy of the light could be calculated using following equation:

$$
E=P S t
$$

Where $P$ is the power density of the light, $S$ and $t$ are the irradiation area $(\mathrm{d}=6 \mathrm{~cm})$ and 
irradiation time. The photothermal conversion efficiency of the BPBBT in nanofibrous mat was calculated following:

$$
\eta=\frac{Q}{E}=c m \Delta T / P S t
$$

As shown in Figure 3B, within $120 \mathrm{~s}$ of irradiation of sunlight, the temperatures of BPBBT nanofiber, CS-1, CS-2 and CS-3 nanofibrous mats reached a maximum value at 40.4, 46.8, 50.3 and $56.1^{\circ} \mathrm{C}$, respectively. According to the equation, their photothermal conversion efficiencies are $0.86 \%, 3.54 \%, 10.15 \%$ and $22.36 \%$, respectively (Figure 3C). Interestingly, the photothermal conversion efficiency of CS-3 is 26 times higher than that of BPBBT nanofiber, solidly implying that the construction of core-shell structure is an efficient strategy to boost the photothermal conversion. Moreover, the nonwoven mat of CS-3 also displayed good durability under extended sunlight irradiation. As shown in Figure 3D, there was no obvious change for the temperature after 9 rounds of irradiation, indicating high stability of these presented core-shell nanofibers. Such durability is a guaranteed for a long working life span the devices that were constructed by the fibers (Figure S4).
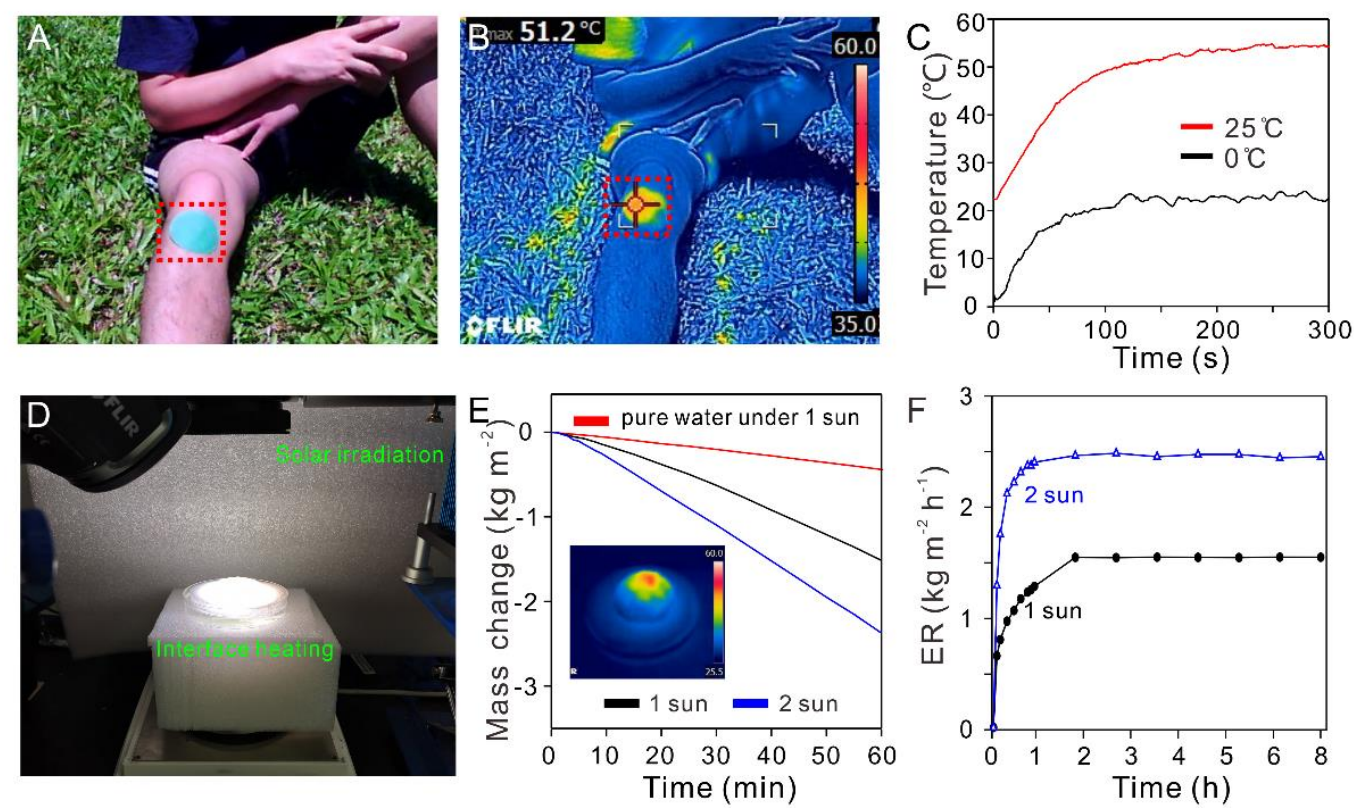

Figure 4. (A) Optical and (B) infrared image of a photothermal patch for warming a volunteer's knee under natural sunlight. (C) The temperature changes of BPBBT CS-3 in varies of ambient 
temperature of 0 and $25^{\circ} \mathrm{C}$ as they were exposed under natural sunlight. (D) Photograph of solar steam generation system. (E) the mass loss of water and (F) Evaporation rate of BPBBT CS-3 over time under different illumination intensity.

We also demonstrated the application of the core-shell nanofiber in photothermal textile and solar steam generation. As shown in digital and infrared images (Figure 4A and B), after a volunteer covering a core-shell nanofibrous patches on the knee under the natural sunlight for one minute, the temperature of the patch reaches at $51.2{ }^{\circ} \mathrm{C}$, which is higher than the around skin and ambient. This result shows that the photothermal patch can be regulated for warming, drug-controlled releasing and therapy. Even the ambient temperature was set at $0{ }^{\circ} \mathrm{C}$. the temperature of BPBBT CS-3 reached at about $24{ }^{\circ} \mathrm{C}$ (Figure 4C), under the irradiation of natural sunlight. The outdoor clothing made of such photothermal materials induced by natural sunlight would be beneficial for human in cold environment. For solar steam generation, the BPBBT CS-3 was placed into solution of $\mathrm{NaBH}_{4}$, the bubbles of $\mathrm{H}_{2}$ trapped into the CS-3 and expanded it to three-dimensional (3D) structure (Figure S5A), which has been reported by previous literature. ${ }^{29,30}$ As shown in Figure 4D, to construct solar steam generation system, the 3D CS-3 was wrapped by foam and then placed into a beaker contained $3.5 \mathrm{wt} \% \mathrm{NaCl}$ water solution (Figure S5B). Under the irradiation of 1 and 2 sun, the temperature of 3D CS-3 reached at 40 and $48{ }^{\circ} \mathrm{C}$, respectively (Figure S6). It was clearly found that the evaporation rates of 3D CS-3 reach constant values after 40 min irradiation (Figure 4E), and the evaporation rate could reach at 1.52 and $2.42 \mathrm{~kg} \mathrm{~m}^{-2} \mathrm{~h}^{-1}$, with the under irradiation of 1 and 2 sun. It was found that the evaporation rate of the 3D CS-3 under 1 and 2 sun of irradiation retains invariant during period of $8 \mathrm{~h}$, which is a favor to sustainable and stable manipulation in daytime (Figure 4F).

To demonstrate the universality of this method, we also examined the feasibility via utilizing other types of photothermal molecules TFM ${ }^{14}$ and TTTMN (Figure S1). ${ }^{31}$ As displayed in Figure S7, after $120 \mathrm{~s}$ irradiation by 1 sun, the temperature of TFM core-shell nanofibrous mat increased to $39.9{ }^{\circ} \mathrm{C}$, which is higher than that of TFM nanofiber $\left(34.4^{\circ} \mathrm{C}\right)$. The same tendency was observed for TTTMN molecules, the temperature of TTTMN core-shell nanofibrous mat 
$\left(46.3{ }^{\circ} \mathrm{C}\right.$ ) is distinctly higher than that of TTTMN nanofiber (about $39{ }^{\circ} \mathrm{C}$ ) (Figure S8). In addition, the great photothermal capability improvement was also verified by the significantly increased photothermal conversion efficiencies of core-shell nanofibers containing TFM or TTTMN, comparing with their nanofiber counterparts (Figure S9). These results demonstrated that the fabrication of core-shell nanofibers by coaxial electrospinning technique is a universal approach to improve the photothermal efficiency of AIEgens in nanofibers.

In summary, coaxial electrospinning technique was utilized for the construction of core-shell nanofibers with AIEgen/oil solution as the core surrounded by PVDF shell layer. The core-shell architecture remarkably endows AIEgens with sufficient intermolecular motions in nanofibers, and thus promotes the non-radiative energy dissipation. Photothermal investigation reveals that the presented core-shell nanofibers can reach up to $22.36 \%$ of photothermal conversion efficiency, which is 26-fold as the non-core-shell counterpart. Under natural sunlight, the BPBBT CS-3 on the skin can be heated at $51{ }^{\circ} \mathrm{C}$, suggesting its application of photothermal patches and/or clothing. It is also exhibited superior capacity of solar steam generation, under irradiation of 1 sun, the evaporation rate reach at $1.52 \mathrm{~kg} \mathrm{~m}^{-2} \mathrm{~h}^{-1}$. Combining with other notable features of the core-shell nanofibers involving AIEgens in terms of high stability and great universality, the established method in this study represents a wonderful protocol to boost photothermal efficiency of nanofibers, and provides a blueprint for the next generation green and zero-carbon emissions photothermal materials for practical applications.

\section{ASSOCIATED CONTENT}

\section{Supporting Information}

The Supporting Information is available free of charge on the ACS Publications website at DOI: Experimental section; structure and ${ }^{1} \mathrm{H}$ NMR spectrum; SEM images of BPBBT pristine nanofiber and CS-1, CS-2 and CS-3; photographs, TEM images, and thermal images of the core-shell nanofibrous mats of CS-1 and CS-2; temperature changes in the mat of BPBBT CS-3 nanofibrous mats upon repeated irradiation by 1 sun; Photograph, TEM and thermal images of 
TFM pristine nanofibrous mat and TFM core-shell nanofibrous mat upon exposure to 1 sun irradiation; Photograph, TEM and thermal images of TTTMN pristine nanofibrous mat and TTTMN core-shell nanofibrous mat upon exposure to 1 sun irradiation; Table of Performance comparison BPBBT pristine nanofibrous mat and BPBBT core-shell (CS) fibrous mats; photography of 3D CS-3 (PDF).

\section{AUTHOR INFORMATION}

Corresponding Author

*Dong Wang - Centre for AIE Research, College of Material Science and Engineering, Shenzhen University, Shenzhen 518061, P. R. China; orcid.org/0000-0001-5137-0771;

E-mail: wangd@szu.edu.cn

*Ben Zhong Tang — Department of Chemistry, The Hong Kong University of Science and Technology, Kowloon, Hong Kong, China; orcid.org/0000-0002-0293-964;

E-mail: tangbenz@ust.hk

\section{Authors}

Haoxuan Li - Centre for AIE Research, College of Material Science and Engineering and Key Laboratory of Optoelectronic Devices and Systems of Ministry of Education and Guangdong Province, College of Optoelectronic Engineering, Shenzhen University, Shenzhen 518061, P. R. China; Department of Chemistry, The Hong Kong University of Science and Technology, Kowloon, Hong Kong, China; orcid.org/0000- 0003-3903-7364

Haifei Wen - Centre for AIE Research, College of Material Science and Engineering, Shenzhen University, Shenzhen 518061, P. R. China

Zhijun Zhang - Centre for AIE Research, College of Material Science and Engineering and Key Laboratory of Optoelectronic Devices and Systems of Ministry of Education and Guangdong Province, College of Optoelectronic Engineering, Shenzhen University, Shenzhen 518061, P. R. China 
Nan Song - Centre for AIE Research, College of Material Science and Engineering and Key Laboratory of Optoelectronic Devices and Systems of Ministry of Education and Guangdong Province, College of Optoelectronic Engineering, Shenzhen University, Shenzhen 518061, P. R. China; Department of Chemistry, The Hong Kong University of Science and Technology, Kowloon, Hong Kong, China

\section{Notes}

The authors declare no competing financial interest.

\section{ACKNOWLEDGMENTS}

Haoxuan Li and Haifei Wen contributed equally to this work. This work was partially supported by the Natural Science Foundation for Distinguished Young Scholars of Guangdong Province (2020B1515020011), the Natural Science Foundation of China (21801169), and the Science and Technology Foundation of Shenzhen City (JCYJ20190808153415062).

\section{REFERENCES}

(1) Anzenbacher, P.; Palacios, M, A. Polymer Nanofibre Junctions of Attolitre Volume Serve as Zeptomole-Scale Chemical Reactors. Nat. Chem. 2009, 11, 80-86

(2) Wu, T.; Li, H.; Xue, J.; Mo, X.; Xia, Y. Photothermal Welding, Melting, and Patterned Expansion of Nonwoven Mats of Polymer Nanofibers for Biomedical and Printing Applications. Angew. Chem. Int. Ed. 2019, 58, 16416-16421.

(3) Li, H.; Zhang, W.; Ding, Q.; Jin, X.; Ke, Q.; Li, Z.; Wang, D.; Huang, C. Facile Strategy for Fabrication of Flexible, Breathable, and Washable Piezoelectric Sensors via Welding of Nanofibers with Multiwalled Carbon Nanotubes (MWCNTs). ACS Appl. Mater. Interfaces 2019, 11, 38023-38030.

(4) Yang, Y.; Yang, X.; Fu, L.; Zou, M.; Cao, A.; Du, Y.; Yuan, Q.; Yan, C. H. Two-Dimensional Flexible Bilayer Janus Membrane for Advanced Photothermal Water Desalination. ACS Energy Lett. 2018, 3, 1165-1171. 
(5) Lu, Y.; Xiao, X.; Fu, J.; Huan, C.; Qi, S.; Zhan, Y.; Zhu, Y.; Xu, G. Novel Smart Textile with Phase Change Materials Encapsulated Core-Sheath Structure Fabricated by Coaxial Electrospinning. Chem. Eng. J. 2019, 355, 532-539.

(6) Xu, Y.; Xu, H.; Zhu, Z.; Hou, H.; Zou, J.; Cui, F.; Liu, D.; Wang, W. A Mechanically Durable, Sustained Corrosion Resistant Photothermal Nanofiber Membrane for Highly Efficient Solar Distillation. J. Mater. Chem. A 2019, 7, 22296-22306.

(7) Lyu, S.; He, Y.; Yao, Y.; Zhang, M.; Wang, Y. Photothermal Clothing for Thermally Preserving Pipeline Transportation of Crude Oil. Adv. Funct. Mater. 2019, 29, 1900703.

(8) Jia, H; Zhu, Y.; Liu, X.; Pan, G.; Gao, G.; Sun, W.; Zhang, X.; Jiang, Y.; Wu, F. Construction of Dually Responsive Nanotransformers with Nanosphere-Nanofiber-Nanosphere Transition for Overcoming the Size Paradox of Anticancer Nanodrugs. ACS Nano 2019, 13, 11781-11792.

(9) Qian, Q.; Wang, J.; Yan, F.; Wang, Y. A Photo-annealing Approach for Building Functional Polymer Layers on Paper. Angew. Chem. Int. Ed. 2014, 53, 4465-4468.

(10) Li, H.; Wen, H.; Li, J.; Huang, J.; Wang, D.; Yang, B. Z. Doping AIE Photothermal Molecule into All-Fiber Aerogel with Self-Pumping Water Function for Efficiency Solar Steam Generation. ACS Appl. Mater. Interfaces 2020, DOI: 10.1021/acsami.0c06181.

(11) Cai, X.; Liu, J.; Liew, W. H.; Duan, Y.; Geng, J.; Thakor, N.; Yao, K.; Liao, L.-D. Liu, B. Organic Molecules with Propeller Structures for Efficient Photoacoustic Imaging and Photothermal Ablation of Cancer Cells. Mater. Chem. Front. 2017, 1, 1556-1562.

(12) Wang, K.; Fan, X.; Zhao, L.; Zhang, X.; Zhang, X.; Li, Z.; Yuan, Q.; Zhang, Q.; Huang, Z.; Xie, W.; Zhang, Y.; Wei, Y. Aggregation Induced Emission Fluorogens Based Nanotheranostics for Targeted and Imaging-Guided Chemo-Photothermal Combination Therapy. Small 2016, 12, 6528-6535.

(13) Zhao, L.; Liu, Y.; Xing, R.; Yan, X. Supramolecular Photothermal Effects: A Promising Mechanism for Efficient Thermal Conversion. Angew. Chem. Int. Ed. 2020, 59, 3793-3801.

(14) Luo, J.; Xie, Z.; Lam, J. W. Y.; Cheng, L.; Qiu, H.; Kwok, H. S.; Zhan, X.; Liu, Y.; Zhu, D.; 
Tang, B. Z. Aggregation-Induced Emission of 1-methyl-1,2,3,4,5-pentaphenylsilole. Chem. Commun. 2001, 18, 1740-1741.

(15) Mei, J.; Leung, N. C.; Kwok, R. T. K.; Lam, J. W. Y.; Tang, B. Z. Aggregation-Induced Emission: Together We Shine, United We Soar! Chem. Rev. 2015, 115, 11718-11940.

(16) Qian, H.; Morgan, E. C.; Erik, H. H; Audrey, W; Matthew, D. L; Ivan, A. Suppression of Kasha's Rule as a Mechanism for Fluorescent Molecular Rotors and Aggregation-Induced Emission. Nat. Chem. 2017, 9, 83-87.

(17) Zhao, Z.; Chen, C.; Wu, W.; Wang, F.; Du, L.; Zhang, X.; Xiong, Y.; He, X; Cai, Y.; Kwok, R. T. K.; Lam, J. W. Y.; Cao, X.; Sun, P.; Philips, D.; Ding, D.; Tang, B. Z. Highly Efficient Photothermal Nanoagent Achieved by Harvesting Energy via Excited-State Intramolecular Motion within Nanoparticles. Nat. Commun. 2019, 10, 768-779.

(18) Wang, D.; Lee, M. M. S.; Xu, W.; Shan, G.; Zheng, X.; Kwok, R. T. K.; Lam, J. W. Y.; Hu, X.; Tang, B. Z. Boosting Non-Radiative Decay to Do Useful Work: Development of A Multi-Modality Theranostic System from AIEgen. Angew. Chem. Int. Ed. 2018, 11, $1510-1519$.

(19) Liu, S.; Li, Y.; Zhang, H.; Zhao, Z.; Lu, X.; Lam, J. W. Y.; Tang, B. Z. Molecular Motion in the Solid State. ACS Mater. Lett. 2019, 1, 425-431.

(20) Wang, J.; Pan, K.; He, Q.; Cao, B. Polyacrylonitrile/Polypyrrole Core/shell Nanofiber Mat for The Removal of Hexavalent Chromium from Aqueous Solution. J. Hazard. Mater. 2013, 244-245, 121-129.

(21) Tsai, P.; Chen, J.; Ercan, E.; Chueh, C.; Tung, S.; Chen, W. Uniform Luminous Perovskite Nanofibers with Color-Tunability and Improved Stability Prepared by One-Step Core/Shell Electrospinning. Small 2018, 14, 1704379.

(22) Wang, C.; Wang, J.; Zeng, L.; Qiao, Z.; Liu, X.; Liu, H.; Zhang, J.; Ding, J. Fabrication of Electrospun Polymer Nanofibers with Diverse Morphologies. Molecules 2019, 24, 834-867.

(23) Song, J.; Wu, X.; Zhang, M.; Liu, C.; Yu, J.; Sun, G.; Si, Y.; Ding, B. Highly Flexible, Core-shell Heterostructured, and Visible-Light-Driven Titaniabased Nanofibrous 
Membranes for Antibiotic Removal and E. coil Inactivation. Chem. Eng. J. 2020, 379, 122269.

(24) Li, D.; Xia, Y. Direct Fabrication of Composite and Ceramic Hollow Nanofibers by Electrospinning. Nano Lett. 2004, 5, 933-938.

(25) Wu, Y.; Chen, C.; Jia, Y.; Wu, J.; Huang, Y.; Wang, L. Review on Electrospun Ultrafine Phase Change Fibers (PCFs) for Thermal Energy Storage. Appl. Energy 2018, 210, 167-181.

(26) Gao, S.; Wei, G.; Zhang, S.; Zheng, B.; Xu, J.; Chen, G.; Li, M.; Song, S.; Fu, W.; Xiao, Z.; Lu, W. Albumin Tailoring Fluorescence and Photothermal Conversion Effect of Near-Infrared-II Fluorophore with Aggregation-induced Emission Characteristics. Nat. Commun. 2019, 10, 2206-2221.

(27) Qian, G.; Dai, B.; Luo, M.; Yu, D.; Zhan, J.; Zhang, Z.; Ma, D.; Wang, Z. Y. Band Gap Tunable, Donor-Acceptor-Donor Charge-Transfer Heteroquinoid-Based Chromophores: Near Infrared Photoluminescence and Electroluminescence. Chem. Mater. 2008, 20, 6208-6216.

(28) Li, J.; Yang, M.; Sun, X.; Yang, X.; Xue, J.; Zhu, C.; Liu, H.; Xia, Y. Micropatterning of the Ferroelectric Phase in a Poly(vinylidene difluoride) Film by Plasmonic Heating with Gold Nanocages. Angew. Chem. Int. Ed. 2016, 55, 13828-13832.

(29) Jiang, J.; Carlson, M. A.; Teusink, M. J.; Wang, H.; MacEwan, M. R.; Xie, J. Expanding Two-Dimensional Electrospun Nanofiber Membranes in the Third Dimension by a Modified Gas-Foaming Technique. ACS Biomater. Sci. Eng. 2015, 1, 991-1001.

(30) Chen, S.; Wang, H.; McCarthy, A.; Yan, Z.; Kim, H. J.; Carlson, M. A.; Xia, Y.; Xie, J. Three-Dimensional Objects Consisting of Hierarchically Assembled Nanofibers with Controlled Alignments for Regenerative Medicine. Nano Lett. 2019, 19, 2059-2065.

(31) Gupta, A.; Ali, A.; Bilic, A.; Gao, M.; Hegedus, K.; Singh, B.; Watkins, S. E.; Wilson, G. J.; Bach, U.; Evans, R. A. Absorption Enhancement of Oligothiophene Dyes through The Use of a Cyanopyridone Acceptor Group in Solution-Processed Organic Solar Cells. Chem. Commun. 2012, 48, 1889-1891. 